\title{
EL LUNFARDO: ¿UN HABLA DE DELINCUENTES QUE QUEDÓ EN EL PASADO?
}

\author{
Daniela Soledad Gonzalez
}

\section{(9) $\odot \Theta \Theta$}

Esta obra está bajo una licencia Creative Commons

Reconocimiento-No Comercial-Sin Obra Derivada 



\title{
EL LUNFARDO: ¿UN HABLA DE DELINCUENTES QUE QUEDÓ EN EL PASADO?
}

\author{
LUNFARDO: DELINQUENT SPEECH LOST IN THE PAST?
}

\author{
Daniela Soledad Gonzalez
}

\begin{abstract}
RESUMEN
En la segunda mitad del siglo XIX se formaron conglomerados alrededor de la ciudad de Buenos Aires cuyos pobladores fueron inmigrantes o porteños de escasos recursos. Este nuevo grupo social creó una especie de subcultura, presente en muchas letras de tangos y en el lenguaje, en el cual hizo su aparición el fenómeno llamado lunfardo. En este trabajo se intentará demostrar que el lunfardo no se limita a ser un habla perteneciente a criminales y que sigue realmente vigente en la actualidad, en el habla cotidiana de los argentinos de todos los niveles sociales. En primer lugar, se hará una breve referencia al estado actual de los estudios sobre el lunfardo; en segundo lugar, se lo definirá; en tercer lugar, se observará en él la presencia de italianismos; en cuarto lugar, se desarrollará brevemente su influencia en la literatura. Luego, se hará un breve comentario de su impronta en el tango; a continuación, se fundamentará la presencia del lunfardo en el habla cotidiana, sobre la base de un corpus de expresiones utilizadas en la actualidad. Finalmente, se presentará una conclusión. Palabras clave: lunfardo, italianismos, tango, habla cotidiana, estratos socioculturales.
\end{abstract}

\begin{abstract}
In the second half of the nineteenth century, conglomerates were formed around Buenos Aires town, whose residents were immigrants or locals with limited resources. This new social group created a kind of subculture, present in many tango lyrics and in the language, where the phenomenon called lunfardo appeared. This paper attempt to demonstrate that the lunfardo is not restrictively a way of speaking belonging to criminals and that it is really in force today, in everyday speech of Argentines of all social levels. First, it will be made a short reference to the current state of studies about lunfardo; secondly, it will be defined; thirdly, the presence of Italianisms in it will be observed; fourthly, its influence on literature will be briefly developed. Then, a short comment about its traces on the tango will be done; below, the presence of lunfardo in everyday speech will be founded on the basis of a corpus of expressions used currently. Finally, a conclusion will be presented.
\end{abstract}

Key words: lunfardo, italianisms, tango, everyday speech, socio-cultural strata.

\section{El estado actual de los estudios sobre el lunfardo}

A pesar de que existen varios diccionarios sobre el lunfardo y otros escritos con aseveraciones sobre este fenómeno, podría afirmarse con Conde (2004, p. 11) que muchas

Lic. Daniela Soledad Gonzalez. Universidad Nacional de Cuyo. Profesora Jefe de Trabajos Prácticos de Morfología y Sintaxis. Becaria de investigación doctoral por CONICET. Argentina.

Correo electrónico: gonzalezdanielasoledad@yahoo.com.ar

Recepción: 22- 02- 2015

Aceptación: 20- 06- 2015 
de las recopilaciones lexicográficas resultan deficientes y que presentan prejuicios que han acompañado a la caracterización del lunfardo por años y que pueden observarse en las discutibles clasificaciones de algunos lexemas como "familiar, popular, delictivo, grosero, etc." (Cfr. Conde, 2004, p. 11). Este tipo de consideraciones son las que revisa Conde en su Diccionario etimológico del lunfardo (2004), en el que presenta un amplio corpus de lexemas. El objetivo de este trabajo es el de actualizar el léxico lunfardo, eliminar pseudolunfardismos ${ }^{1}$ y ofrecer la mayor cantidad posible de etimologías de las palabras presentadas.

Una entidad crucial para el estudio riguroso del lunfardo es la que se fundó en 1962 con el fin de hacer un seguimiento de la evolución del mismo: la Academia Porteña del Lunfardo. Es una institución privada, sin fines de lucro (ONG). La iniciativa fue fruto de las ideas de José Gobello, Nicolás Olivari y Amaro Villanueva. Gobello, acompañado por Luis Soler Cañas y León Benarós, convocó a un grupo de escritores y periodistas para crear este instituto destinado al estudio del habla popular. A lo largo de su existencia, ha editado libros, folletos y artículos. Además, ha organizado una biblioteca especializada en temas referidos a Buenos Aires y un salón de actos.

En el año 2004, María Luján Picabea publicó una noticia en el diario Clarín titulada Lunfardo siglo XXI. La lengua de los porteños, del arrabal al shopping. En ella, señala algunos estudios actuales sobre "los modos de decir de los jóvenes, que se vuelcan en letras de rock, cumbia o bailanta y se reproducen y legitiman en los programas de radio y $\mathrm{TV}$, las revistas, y los diarios, pertenecen al lunfardo: el Diccionario etimológico del lunfardo, de Conde, el Novísimo diccionario lunfardo (Corregidor, 2004), de José Gobello y Marcelo Oliveri, y Tangueces y lunfardismos del rock argentino (Corregidor, 2001), de los mismos autores" (Picabea, 2004, p. 1). Otro estudio importante es el de Héctor E. Musa, autor del Calepino. Lunfoargentino (2005). En este trabajo se puede encontrar, además de una recopilación de un vasto número de palabras y expresiones lunfardas, una explicación de los procedimientos de creación de las mismas (Cfr. Musa, 2005, pp. 39-46) y algunas consideraciones sobre el fenómeno del lunfardo en general. Muchas de las entradas presentes en esta obra están acompañadas de fragmentos de letras de tangos y otras producciones culturales (v. gr., obras de teatro, historietas) que ejemplifican su uso.

\section{2. ¿Qué es el lunfardo?}

José Esteban (2002, pp. 39-40) afirma que no es fácil definir el lunfardo. Señala que no existe una única definición de este fenómeno: "Los puristas dicen que es el lenguaje de las cárceles y los delincuentes y desde luego no está bien visto por la clasista sociedad bonaerense, aunque todo el mundo lo utiliza, lo entiende y lo fomenta". En cuanto a la concepción del lunfardo como habla de delincuentes, Esteban (2002, p. 43) añade que se trata de un "lenguaje críptico que manejaban los reclusos y mediante el que lograban burlar a los guardianes, dando vueltas y revueltas a un limitado número de palabras a los que atribuían significados muy dispares y siempre alejados de la acepción original". Es necesario completar esta definición.

Es atractiva la manera de definir el lunfardo utilizada por Oscar Conde en su conferencia sobre la relación entre el lunfardo y el cocoliche, presentada en el año 2009. Comienza por decir lo que el lunfardo no es. Entre estas definiciones negativas se encuentra la siguiente:

Por décadas - en un error del que ni siquiera Borges estuvo al margen - se consideró al lunfardo como un léxico de la delincuencia (...) El hecho de que el término lunfardo significara en su origen 'ladrón' llevó a 
conclusiones erróneas a los primeros que se acercaron a estudiar el fenómeno. Pero el lunfardo no es - ni lo fue nunca - un vocabulario delictivo [...]. Un artículo de Juan Piaggio de 1887 ya demuestra el error, al presentar a dos jóvenes humildes - pero no delincuentes -, chamuyando en lunfa, y utilizando voces como tano, chucho, batuque, morfi, escabiar y vento, todas ellas perdurables hasta hoy.

Esto no significa que no haya un gran caudal de léxico procedente del ámbito del delito en el lunfardo. De hecho, las dos primeras acepciones de la palabra que Conde (2010, p. 205) presenta en su diccionario son: 1) 'ratero, ladrón' y 2) ‘jerga del ladrón porteño'.2 Pero no se debe limitar la riqueza del lunfardo, pues abarca muchos otros ámbitos de la vida cotidiana, como podrá observarse en el apartado 7 (siete) de este trabajo.

Continúa Conde (2009), aclarando que el lunfardo tampoco es un idioma:

\begin{abstract}
El lunfardo no es un idioma, porque las palabras que lo componen son esencialmente verbos, sustantivos y adjetivos, de manera tal que carece de pronombres, preposiciones, conjunciones - y prácticamente de adverbios - y porque utiliza la misma sintaxis y los mismos procedimientos flexionales que el castellano. No es posible hablar completamente en lunfardo, sino a lo sumo hablar con lunfardo.
\end{abstract}

Franchi (2008) amplía esta explicación "El lunfardo no es un idioma en sí mismo, ya que sus sustantivos, adverbios y verbos no son suficientes para lograr una sintaxis completa, sino que deben ser utilizados intercambiándose en una frase por una palabra que sea su sinónimo en español". ${ }^{3}$ Como el mismo autor aclara luego, si bien el lunfardo es propiamente un fenómeno que afecta al léxico, conlleva algunos cambios gramaticales. Implica, en ocasiones, una modificación de la sintaxis castellana estándar. El lunfardo podría considerarse, entonces, un argot, sin olvidar la complejidad de este fenómeno.

En relación con esto, la definición negativa de Conde (2009) que se viene comentando añade que el lunfardo tampoco es un dialecto, pues un dialecto es una variedad regional de una lengua. Existe un dialecto rioplatense o porteño de la lengua española, pero eso implica la confluencia de distintos elementos además de aquellos que pertenecen al campo lexical: una fonética determinada, un determinado uso de los pronombres (vos podés y no vos puedes, por ejemplo).

Conde (2009) cree que el lunfardo debe ser entendido como un "modo de expresión popular", un "repertorio léxico integrado por palabras y expresiones de diverso origen, utilizadas en alternancia con las del español estándar y difundido transversalmente en todas las capas sociales y centros urbanos de la Argentina". Si se complementa esta definición con la que el mismo autor da en su diccionario (Cfr. Conde, 2010, p. 205), se puede realizar la siguiente caracterización del lunfardo:

a. Se trata de un "habla" o "modo de expresión".

b. Fue empleada originariamente en la ciudad de Buenos Aires y sus alrededores por la gente de clase baja. Luego, fue difundido por todas las capas sociales de la Argentina.

c. Se trata esencialmente de un repertorio de voces y expresiones populares formadas a partir de lexemas aportados por la inmigración y la jerga ladronil.

d. Se lo utiliza en alternancia con el vocabulario del español estándar.

\title{
3. Presencia de italianismos en el lunfardo
}

Como afirma Casas (1991), el lunfardo está plagado en toda su extensión de un enorme caudal léxico procedente de los países de origen de los inmigrantes llegados a Argentina en el 
siglo XIX. Conde (2009) desarrolla el amplio espectro de los inmigrantes que influyeron en el lunfardo: afronegrismos, lusismos, anglicismos, aborigenismos, etc. No duda en indicar que uno de los aportes más definitorios para la formación del lunfardo fueron las lenguas itálicas.

Sobre este particular de la inmigración italiana, Casas (1991, p. 28) afirma:

\begin{abstract}
Entre 1821 y 1932, llegaron a estas tierras cincuenta y un millones de europeos. De ellos, diez millones eran individuos procedentes de la península Itálica. Merece la pena destacar la riqueza dialectal de Italia. Esta característica es la que ha posibilitado directamente la existencia de términos en el lunfardo procedentes de las regiones más variadas de Italia. Así, encontramos préstamos del genovés, del véneto, del milanés o del piamontés.
\end{abstract}

Es igualmente interesante destacar con Casas (1991) que la procedencia social de la mayor parte de los inmigrantes correspondía a las capas sociales más bajas. Además, la gran cantidad de extranjeros llegados a esta zona se instaló en la ciudad de Buenos Aires. Todas estas circunstancias llevaron al hacinamiento y a la proliferación de la delincuencia.

Casas hace una interesante recopilación de italianismos, de los cuales se extraerán solo tres como botón de muestra. El primero es atenti ('¡Cuidado!’), una interjección que se supone que podría provenir del italiano attento o attenti. ${ }^{4} \mathrm{El}$ segundo ejemplo es escabiar ('tomar bebidas alcohólicas'). Es un verbo derivado del sustantivo escabio, que parece provenir del italiano jergal, de alguna de las siguientes formas de origen: scabbia, scabbio, scabbi y scabi. Estos términos significan 'vino'. En lunfardo se produce una extensión del significado y designa a la 'bebida alcohólica en general'. De este sustantivo, como ya se ha señalado, se deriva el verbo escabiar, con el significado de 'beber vino u otras bebidas alcohólicas' y 'emborracharse'.

El último ejemplo es el lexema bacán ('hombre adinerado que mantiene una mujer'). Se acepta, en general, un origen genovés para esta palabra. Se documentan dos formas similares en este dialecto: bacan y baccan. El sentido que la palabra tiene en genovés es el de 'amo o dueño'. Es una de las voces lunfardas que se suponen más antiguas y todavía pervive hoy en el habla de la calle. Pasa a formar parte del lunfardo con el significado de 'dueño de una mujer' o también 'concubinario'. De aquí pudo trasladarse la significación fácilmente al sentido actual de 'hombre rico, adinerado'. Y a usarse como adjetivo, 'lujoso'. Únicamente en el tango aparece con el significado más genérico de 'hombre'.

Oscar Conde (2009) afirma que el lunfardo y el cocoliche son dos fenómenos nacidos en el Río de la Plata en la segunda mitad del siglo XIX, relacionados entre sí, pero diversos. Citando a Gobello y Oliveri (2005, p. 15), señala que "el primer esfuerzo para hacerse comprender derivó a cocoliche, lenguaje de transición. Lo hablaban los inmigrantes. El segundo esfuerzo, el de los hijos de los inmigrantes, derivó a lunfardo". Añade que el uso del cocoliche literario se fue desvaneciendo con el grotesco durante la década de 1930, a pesar de que el cocoliche cotidiano siguió escuchándose algunos años más. En cambio, el lunfardo, se fue despegando de las influencias de las lenguas de la península itálica y comenzó a generar nuevas palabras a partir de recursos ya conocidos, como el vesre, la metaforización, la sinécdoque y la ampliación o restricción de significado de voces españolas ya existentes.

\title{
4. El lunfardo en la literatura
}

A comienzos del siglo pasado, jugaron un importante papel en la difusión del lunfardo las revistas ilustradas (Caras y Caretas, PBT, Fray Mocho), que dieron lugar a viñetas y crónicas en cuya prosa el lunfardo comenzó a tener presencia como recurso expresivo y caracterizador 
de los personajes. Más adelante, el lunfardo formó parte también de las aguafuertes, de las páginas de humor y de algunas otras secciones de las revistas (v. gr., policiales, deportes), lo cual muestra cómo fue siendo incorporado progresivamente en la lengua oficial.

Con la popularización literaria del lunfardo se produjo una progresiva reducción de las frases gauchescas y las expresiones cocoliches o cocolichescas. ${ }^{5}$ Eran los tiempos de Edmundo Montagne, Santiago Dallegri, Félix Lima y Juan Francisco Palermo (Cfr. Conde, 2010, p. 233).

La primera novela lunfarda, según Conde (2010, p. 233) fue La muerte del pibe Oscar, publicada en 1926 en forma de libro, aunque su autor, Luis Villamayor, la dio a conocer por entregas en 1913, en la revista Sherlock Holmes. A esta obra siguieron El deschave (1965), editada por Arturo Cerretani; El vaciadero (1971), de Julián Centeya; Jeringa (1975) y Despertá, Jeringa (1985), estas últimas escritas por Jorge Montes. Para ilustrar el estilo de estas obras, puede considerarse el siguiente fragmento de Jeringa:

\footnotetext{
Vivíamos en un inquilinato de la caye Pichincha frente al Mercado Spinetto. Era una saca vieja de planta baja, media cuadra de lunga, que se alquilaba por habitaciones unidas como ristra de ajo. En el escalafón de la mishiadura, el inquilinato subía apenas un tablón más arriba del conventiyo. Su trajinar de gente armaba un desfile perpetuo, un constante entrar y salir. Los patios resultaban una caye más del barrio y por eyas patruyaba mercando cuanto vendedor andaba suelto por la rúa. Y como los mangantes caían en tropel, parecía Florida y Corrientes al piantar los laburantes de las oficinas. Sólo faltaban un par de chantas parados entre el segundo o tercer corredor vendiendo bayenitas o la Guía de la Ciudad de Buenos Aires y era «cartón yeno» (Montes, 1975, p. 17)
}

En este pasaje puede observarse que el narrador utiliza palabras lunfardas como lunga ('larga'), mishiadura ('indigencia'), mercar ('comprar'), rúa ('calle'), mangante ('ladrón, pillo'), piantar ('huir, robar, echar a alguien'), laburante ('trabajar') y chanta ('persona poco creíble'). Además, se marca la pronunciación yeísta rehilada porteña de la ye y la elle colocando y en palabras como caye y conventiyo, recurso que, junto al uso del lunfardo, refleja el habla de la época y caracteriza de modo realista a los personajes.

Otro autor ineludible de la literatura lunfardesca fue Miguel Ángel Bavio Esquiú, que utilizó el pseudónimo de Juan Mondiola para forjar un personaje canchero que describe la realidad de los años cuarenta y cincuenta con extraordinaria agudeza y un tono "levemente sobrador" (Conde, 2010, p. 234). También el lunfardo tuvo una presencia innegable en el sainete, cuyo autor más significativo fue Alberto Vacarezza. En su obra Tu cuna fue un conventillo (1920), en el cuadro dos, un criollo aconseja a un italiano cómo seducir a una mujer; en un solo diálogo se refiere a la mujer como el mosaico, la percha, el rombo, la nami (verse por mina), el dulce, la percanta y la bandeja, lo cual muestra a las claras - además del dominio del lunfardo que poseía Vacarezza - la intención de reflejar el habla de entonces presente en la literatura de la época, sobre todo en el género teatral. En la pluma de Vacarezza, el recurso al lunfardo, la invención de palabras, así como el uso de la lengua coloquial en general, sirven como recursos de humor y ponen el acento en determinadas características de los personajes.

Para dar un breve muestrario de palabras lunfardas en la literatura, muchas de las cuales se han mantenido hasta hoy y se usan en diversos estratos socioculturales, se han extraído las siguientes expresiones de la obra El conventillo de la Paloma, de Vacarezza: chapársela conmigo ('agarrársela conmigo', hoy en día con el significado de 'besarse apasionadamente'), grela ('mujer'), batir ('decir'), aparato (en sentido despectivo, 'mujer'; hoy en día usado con el significado de 'bufón o extravagante'), carcajear ('reír a carcajadas'), me he ido al mazo sin orejiar ('me he salido del juego sin mirar las cartas'), pinta abacanada (se dice de alguien vestido y arreglado como pudiente), rajá ('andate'), emplichándose ('vistiéndose con ropas 
formales'), bulín, yapa ('segunda oportunidad'), me has madrugado ('me embromaste'), perdé cuidao ('no te preocupés'), rechifle ('locura o perturbación'; hoy se usa más chifle y sus derivados), apoliyar ('dormir').

Dos grandes autores conocedores del lunfardo, que lo utilizaron en sus obras, fueron Borges y Roberto Arlt. En cuanto a Borges, puede decirse mucho. Solo se hará referencia al comentario que sobre el mismo hace Conde (2010, p. 239): "posiblemente el mayor de los detractores del lunfardo, no lo ignoraba en absoluto". En cambio, sobre Arlt se puede decir que no solo no ignoraba el habla porteña popular, sino que la consagró y defendió en su obra, como puede observarse en el siguiente fragmento:

\footnotetext{
Y yo tengo esta debilidad: la de creer que el idioma de nuestras calles, el idioma en que conversamos usted y yo en el café, en la oficina, en nuestro trato íntimo, es el verdadero. ¿Qué yo hablando de cosas elevadas no debía emplear estos términos? Créanme. Ningún escritor sincero puede deshonrarse ni se rebaja por tratar temas populares y con el léxico del pueblo. Lo que es hoy caló, mañana se convierte en idioma oficializado. Además, hay algo más importante que el idioma, y son las cosas que se dicen (Arlt, 1998, pp. 371-373)
}

Para un estudio más detallado de la presencia del lunfardo en la obra de Arlt, puede consultarse Pitkowski (2008), cuyo artículo se centra en los vocablos rajá y turrito, presentes en la obra Los siete locos (1929). Existen muchos otros autores renombrados que utilizaron el lunfardo en sus producciones. Solo se hará una lista acotada de algunos de ellos: Leopoldo Marechal, Manuel Gálvez, Ernesto Sábato, Julio Cortázar y Roberto Fontanarrosa. ${ }^{7}$

No hay que olvidar que, negando el lenguaje culto y dándole curso y legitimidad al lunfardo, el tango se convirtió en el medio más adecuado para que el lenguaje lunfardesco creciera en sus posibilidades expresivas. Sobre este particular se realizan algunas consideraciones en el apartado siguiente.

\section{El lunfardo en el tango}

Como ya se ha expresado, en un primer momento el uso del lunfardo estuvo fuertemente marcado sociolingüísticamente, pero con el paso del tiempo se dio su "oficialización", para usar el concepto expresado por Arlt en la cita del apartado anterior. Como es sabido, el tango contribuyó de modo decisivo a la "expansión del lunfardo en forma transversal, difundiéndolo en todos los sectores sociales" (Cfr. Conde, 2009).

Sería interminable la lista de obras (lunfardas o lunfardescas) que se podrían enumerar como ejemplos de esto. ${ }^{8}$ Solo se presentará un fragmento de la letra del tango Mano a mano, escrito por Celedonio Flores en 1920:

\footnotetext{
Se dio juego de remanye cuando vos, pobre percanta gambeteabas la pobreza en la casa de pensión. Hoy sos toda una bacana, la vida te ríe y canta, los morlacos del otario los tirás a la marchanta como juega el gato maula con el mísero ratón.
}

En este tango, un criollo se dirige a la mujer que antes amó y que ahora tiene amoríos con otro hombre por su dinero. Le recuerda que su origen es pobre, aunque ahora tenga una buena situación económica. Agrega que, por mucho dinero que tenga, no hallará nunca la felicidad y que, por ello, se encuentran "mano a mano" y no le guarda rencor por haberlo abandonado.

Como señala Franchi (2008, p. 1), "la fuerza expresiva de estas líneas depende no sólo de la maestría poética de Celedonio Flores, sino del correcto intercambio de las palabras 
lunfardas y la utilización (o no) de las del idioma español". Acompaña a este fragmento un glosario con los términos lunfardos presentes en él, que se coloca a continuación.

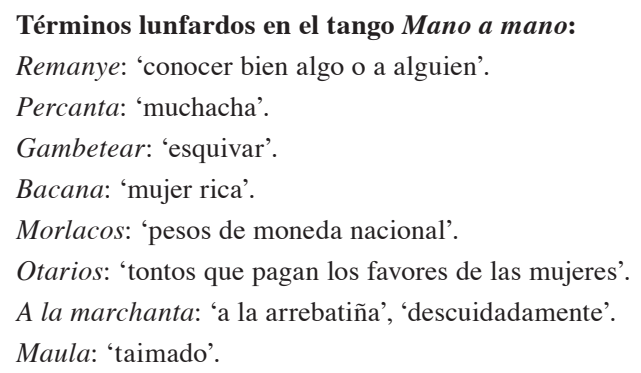

Algunos de estos términos como otario y gambetear se encuentran en uso en la actualidad; otros, como morlaco, parecen haber caído en desuso. Lo cierto es que en el siglo XX tenían plena vigencia y ampliaron su campo de acción, al tiempo que se iba generalizando la aceptación del lunfardo y se lo iba incorporando en la lengua estándar.

\section{El lunfardo en la vida cotidiana en la actualidad}

\section{1 ¿Se oyen palabras lunfardas en la vida cotidiana en la actualidad?}

Según Oliveto (2010), durante los años veinte, el lunfardo adquirió notoria relevancia en el marco de las discusiones sobre el idioma nacional porque este vocabulario, de uso exclusivo de los sectores populares, se había convertido en un componente idiomático de casi todo Buenos Aires. Añade: "La extensión en la utilización del lunfardo preocupa a algunos filólogos, periodistas y escritores quienes piensan que ese vocabulario está llamado a desempeñar un rol fundamental en la formación de un idioma nacional, desprendido del castellano heredado de España"9 (Oliveto, 2010, p. 2).

En cuanto a la situación actual del lunfardo, Picabea, en la ya mencionada noticia aparecida en el diario Clarín en el año 2004, sostiene que, aunque en la conciencia de muchos argentinos no sea un hecho evidente que el lunfardo está muy vivo en el habla de todos, esto es innegable. La autora cita a Conde, que se pregunta "Si el lunfardo ya no existe, ¿cómo llamamos a las formas de hablar de los adolescentes cuando dicen, por ejemplo, estoy al palo, te zarpaste, o ese chabón está de la cabeza?" (Picabea, 2004, p. 1), expresiones que ningún argentino puede negar que ha dicho u oído más de una vez.

Al hecho de que los argentinos hablen con un enorme caudal de palabras provenientes del lunfardo de los siglos XIX y XX, Picabea añade que muchas palabras son tomadas en la actualidad de los dominios del rock, el mundo de las drogas y la "estética villera" para ser utilizadas como lunfardo de este siglo. A estos ámbitos proveedores de lunfardo pueden agregarse otros señalados por Conde (2004, p. 14; 2010, p. 232): el fútbol, los oficios y profesiones, las llamadas tribus urbanas, el psicoanálisis, el boxeo, el automovilismo, la radio, la televisión e internet. ${ }^{10}$ Algunas de las palabras nuevas pertenecientes a los ámbitos mencionados son flash ('sensación súbita de bienestar'), bajón ('depresión que sigue al efecto de una droga'), heavy ('pesado'), gato ('ladrón'), y escrachar ('hacer pública alguna información de alguien en contra de su voluntad').

Para resumir lo dicho hasta aquí, en los casos en los que se crean palabras para hablar en clave sobre temas tabúes (v. gr., la droga) ya no se trata de un lunfardo en etapa final de 
fosilización, en la que el hablante no tiene conciencia de hablar lunfardo, sino de un lunfardo en fase inicial, aquella en la que se reconoce que la palabra es una innovación.

Este nuevo tipo de lunfardo podría ser caracterizado principalmente por la coloquialidad y el encriptamiento de sus expresiones, rasgos estos por los que se lo utilizaría en un sector reducido de la sociedad, socialmente marcado. No obstante, este fenómeno va ampliando ya su espectro de influencia a otros sectores sociales, como sucedió con el lunfardo de los siglos XIX y XX. Tanto las características del lunfardo siglo XXI como el proceso de extensión social del mismo son asuntos que merecen ser estudiados con mayor profundidad.

\section{2 Algunas palabras lunfardas utilizadas en la vida cotidiana}

En este apartado se presenta un corpus de veinte vocablos lunfardos utilizados en la Argentina en la vida cotidiana. Para su compilación, se han consultado fundamentalmente los estudios de Esteban (2002) y Conde (2004). Téngase en cuenta que, de los lexemas presentados por José Esteban se han seleccionado solo los que se hallaron también en el diccionario de Conde o el de Musa, pues el estudio de Esteban registra de modo general palabras que constituyen variantes del español de América respecto del español de España, algunas de las cuales no son porteñas (v. gr. carne ${ }^{11}$ durazno), razón por la cual podrían ubicarse fuera del ámbito del lunfardo "estricto", tal como se lo ha definido en el apartado tres.

A continuación se exhibe una acotada lista de palabras oídas y pronunciadas con naturalidad por la autora del presente trabajo en la vida cotidiana, que provienen del lunfardo. A la derecha de cada lexema se colocan su significado y referencias bibliográficas sobre estudios donde se encuentran registradas.

Acomodo: 'posición de privilegio lograda gracias a otro' (Cfr. Conde, 2004, p. 37).

Afanar: 'hurtar, robar' (Cfr. Conde, 2004, p. 38).

Ave negra o avenegra: 'abogado' (Cfr. Esteban, 2002, p. 43, Conde, 2004, p. 52 y Musa, 2005, tomo 1, p. 161).

Bardear: 'causar desorden, confusión', 'hacer mal las cosas', 'increpar, provocar', 'molestar, agredir verbalmente o burlarse de alguien' (Cfr. Conde, 2004, p. 58 y Musa, 2005, tomo 1, p. 195).

Bife: 'filet' (Cfr. Esteban, 2002, p. 40 y Conde, 2004, p. 63).

Bolacear/bolasear: 'mentir, inventar una historia o bolazo' (Cfr. Conde, 2004, p. 65 y Musa, 2005, tomo 1, p. 236).

Bondi: 'tranvía, ${ }^{12}$ ómnibus' (Cfr. Conde, 2004, p. 69).

Bronca: 'enojo fuerte' (Cfr. Conde, 2004, p. 71).

Chamuyar/chamullar: 'hablar en tono confidencial y persuasivo' (Cfr. Esteban, 2002,p. 44, Conde, 2004, p. 96 y Musa, 2005, tomo 1, p. 377).

Coger: 'realizar el coito' (Cfr. Esteban, 2002, pp. 41 y 45, Conde, 2004, p. 108 y Musa, 2005, tomo 1, p. 447).

Concha: 'aparato genital masculino' (Cfr. Esteban, 2002, pp. 41 y 45; Conde, 2004, p. 112).

Cuento: ‘embuste, engaño' (Cfr. Conde, 2004, p. 120).

Curro: 'estafa, fraude' (Cfr. Esteban, 2002, p. 45, Conde, 2004, p. 122 y Musa, 2005, tomo 1, p. 506).

Curtir: 'castigar con azotes', 'realizar el coito' (Cfr. Conde, 2004, pp. 122-123).

Cybercafé (abrevido, cyber): 'bar donde se ofrece conexión a internet' (Cfr. Conde, 2004, p. 123).

Embole: 'molestia, aburrimiento' (Cfr. Conde, 2004, p. 136 y Musa, 2005, tomo 2, p. 23).

Emplicharse: 'vestir, generalmente con elegancia' (Cfr. Conde, 2004, p. 138 y Musa, 2005, tomo 2, p. 37).

En la loma de los quinotos: 'muy lejos' (Cfr. Esteban, 2002, p. 39).

Gauchada: 'servicio o favor ocasional prestado con buena disposición (Cfr. Conde, 2004, p. 171).

Groncho: ‘de baja condición, ordinario' (Cfr. Conde, 2004, p. 176).

Hinchar las guindas/las pelotas/los huevos: 'incomodar, fastidiar' (Cfr. Conde, 2004, p. 183). 
Impás: 'suspensión momentánea en el desarrollo de una actividad' (Cfr. Conde, 2004, p. 185).

Mina: 'mujer' (Cfr. Conde, 2004, p. 221).

Muere (en la expresión ir al muere): 'muerte' (Cfr. Conde, 2004, p. 226).

Partusa/partuza: 'orgía', 'fiesta'13 (Cfr. Conde, 2004, p. 246). ${ }^{14}$

Perejil: 'tonto', 'persona de baja jerarquía en una institución' (Cfr. Conde, 2004, p. 254).

Ratearse: 'faltar a clase o al trabajo' (Cfr. Conde, 2004, p. 278).

Tildarse: 'quedarse quieto, estar súbitamente desganado' (Cfr. Conde, 2004, p. 300).

Viejo: 'padre' (Cfr. Esteban, 2002, p. 43 y Conde, 2004, p. 315).

Zafar: 'desligarse de responsabilidades', 'salir victorioso de una situación complicada' (Cfr. Conde, 2004, p. 325).

\subsection{Una muestra acotada de los significados asignados a palabras lunfardas por dos hablantes pertenecientes a sociolectos diversos}

En este apartado se referirá brevemente el resultado de una entrevista realizada a dos hablantes pertenecientes a sociolectos distintos. ${ }^{15} \mathrm{El}$ primero, un hombre adulto, culto, docente de nivel universitario, de posición económica media. El segundo, una mujer adulta, de cultura media-baja, ama de casa, también de posición económica media.

Se les preguntó qué significados tenían para ellos cinco palabras lunfardas: biaba, cachar, cafishio/cafisio/cafisho, calentarse y morfar. En primer lugar, se presenta un repaso de las definiciones que de ellas da Conde (2004):

Biaba: 'golpe, paliza, derrota en una competencia' (Cfr. Conde, 2004, p. 62).

Cachar: 'asir, agarrar', 'sorprender a alguien, descubrirlo', 'burlarse de una persona' (Cfr. Conde, 2004,

p. 77).

Cafishio: 'proxeneta, rufián', ‘elegante, distinguido’ (Cfr. Conde, 2004, p. 79).

Calentarse: 'preocuparse'. Del esp., calentarse: 'excitarse sexualmente, enfervorizarse en una disputa' (Cfr. Conde, 2004, p. 81).

Morfar: 'comer'. En el fútbol, 'retener un jugador excesivamente la pelota' (Cfr. Conde, 2004, p. 224). ${ }^{16}$

En la siguiente tabla se presenta una contrastación de las respuestas obtenidas de los hablantes:

Tabla 1. Comparación de las representaciones mentales sobre palabras lunfardas presentes en dos hablantes de sociolectos distintos

\begin{tabular}{|c|c|c|}
\hline $\begin{array}{c}\text { Palabra } \\
\text { lunfarda }\end{array}$ & Hablante 1 & Hablante 2 \\
\hline Biaba & 'Golpiza, castigo' & -- \\
\hline Cachar & 'Bromear, engañar' & 'Hacer una broma' \\
\hline Cafishio & 'Descubrir una intención o significado' & 'Proxeneta' \\
& $\begin{array}{c}\text { 'Persona a la que le gusta poco trabajar y } \\
\text { vive del trabajo de los demás' }\end{array}$ & $\begin{array}{c}\text { 'Los mujeres' } \\
\text { las }\end{array}$ \\
\hline Calentarse & 'Sentir ira' & 'Excitarse' \\
\hline Morfar & 'Comer grotescamente' & 'Comer' \\
\hline
\end{tabular}


Como puede observarse en el cuadro, el hablante 1 posee una mayor cantidad de representaciones mentales acerca de las palabras presentadas. El hablante 2, además, desconocía una de ellas: biaba. Lo interesante acerca del conocimiento de las palabras presente en el hablante 2 es que registra como únicas las acepciones más etimológicas de las palabras, v. gr., 'proxeneta' para cafishio. Finalmente, se destaca el hecho de que en los dos hablantes, procedentes de sociolectos distintos, existe un conocimiento bastante amplio sobre palabras lunfardas.

\section{Conclusión}

El lunfardo es un fenómeno complejo, difícil de definir. No es un idioma, sino un argot, pues se trata fundamentalmente de un repertorio léxico. Tampoco es un modo de expresión exclusivo del ámbito de la delincuencia. Es un habla coloquial que nació en las capas sociales bajas de la ciudad de Buenos Aires, fundamentalmente de la apropiación de palabras que trajeron los inmigrantes llegados en el siglo XIX, muchas de ellas pertenecientes al dominio de los ladrones y proxenetas. En la actualidad, se halla extendido en el español de Argentina, en todos los estratos socioculturales. Esto pudo advertirse en los resultados de la breve entrevista referida en el apartado 6. 3 de este trabajo.

Ivonne Bordelois (2006, p. 119) afirma que "las lenguas no se presentan como precipitados uniformes de un mismo tipo: antes bien, son muestrarios de variedades diversas y dialogantes y sus riquezas en el transcurso cotidiano de la vida social". Como pudo observarse a lo largo de este trabajo, el habla cotidiana se halla impregnada de palabras y expresiones lunfardas, así como convive con otras variantes del español estándar, que enriquecen el habla de los argentinos. La riqueza del lunfardo es innegable, pues sirve para los más variados ámbitos de la vida y presenta una gran fuerza expresiva. Considérese, por ejemplo, la diferencia entre la palabra mucho y la expresión adverbial lunfarda a rolete (Cfr. Musa, 2005, tomo 1, p. 62).

Es necesario destacar, además - como dice Esteban (2002, p. 43) - que

el lunfardo, el porteño, el canero o el arrabalero, como todo lenguaje nacido y criado en plena calle, evolucionan a gran velocidad a lo largo del tiempo, pero, dicen los expertos, en su evolución, se asimilan nuevos vocablos, pero no se eliminan los anteriores, ${ }^{17}$ con lo que su crecimiento es imparable

Al lunfardo fosilizado se le suman continuamente nuevas palabras tomadas de otros ámbitos sociales marcados, que van haciéndose de uso general paulatinamente. El lunfardo no solo es inevitable, sino que también es permanente, lo cual debiera ser para todos un hecho reconfortante y no vergonzante, pues, como dice Bordelois (2006, p. 135): "el lunfardo es un testimonio de la oreja atenta y receptiva de los argentinos".

\section{Notas}

1. Sorprendentemente, algunos de los enumerados por Conde (2004, p. 15) son: espichar ('morir'), fiambre ('cadáver'), plomo ('persona pesada, molesta'), pollo ('escupitajo') y guita ('dinero').

2. Otro factor influyente en la concepción del lunfardo como un habla de delincuentes es señalada por Conde (2009): "La palabra francesa argot, atestiguada recién en 1634, parece ser una deformación de jargon - literalmente 'jerga' -, término que designó en su origen a cierto vocabulario que corría entre bandoleros, mendigos y vendedores ambulantes en la Francia de los siglos XV y XVI. Llamativamente es esta palabra la que la lingüística contemporánea ha adoptado para denominar a ciertos fenómenos como el que nos ocupa".

3. Franchi ejemplifica esto de la siguiente manera: "Qué lindo sombrero podría traducirse como qué lindo funyi, utilizando uno de los términos lunfardos más conocidos. En principio se supone que debería ser 
algo tan sencillo como eso, sin embargo al acabar de escribir la frase precedente descubro que hay algo más que había pasado por alto hasta este momento: una frase lunfarda no requiere sólo del intercambio de una palabra del español por su sinónimo lunfardo, sino también la no utilización de ciertas otras (...) [El lunfardo] Debe ser recio, cortante, duro. Un guapo que se precie de tal no utiliza la palabra lindo; supongo que la traducción correcta de la frase anterior deberá ser, a lo sumo qué buen funyi".

4. Casas se apoya en la autoridad de Sala y Gobello para afirmar esta procedencia etimológica (Cfr. Casas, 1991, p. 30).

5. Con esta diferencia entre los adjetivos cocoliche y cocolichesco -que luego se repetirá con los términos lunfardo y lunfardesco- se quiere señalar que una palabra es 'cocoliche' o 'al modo del cocoliche', respectivamente.

6. Sobre la caracterización de Borges como "detractor del lunfardo", cfr. Oliveto (2010, p. 4).

7. Para mayor información sobre el conocimiento del lunfardo que manifiestan estos autores, cfr. Conde (2010, pp. 242-243).

8. Para más ejemplos de tangos lunfardos o palabras lunfardas presentes en tangos, consultar Conde, 2010, pp. 236-239.

9. Para una profundización en el asunto de los debates sobre la lengua y la literatura de aquella época (con protagonismo del lunfardo), leer el artículo completo de Oliveto (2010).

10. Sobre este particular, cfr. Bordelois (2006, pp. 131-152).

11. Como aclara el autor, "[En España y otros países] en el epígrafe carne nos encontramos con entrecot, solomillo, cordero asado, chuletas de cerdo, estofado de perdiz y ancas de rana. Pues bien, en la República Argentina el concepto de carne es mucho más limitado; carne, lo que se dice carne es el ternero de menos de un año" (Esteban, 2002, p. 40).

12. La palabra bondi proviene del portugués bonde, que significa 'tranvía'. Es una voz nacida en Río de Janeiro cuando se instalaron los tranvías. En ese momento, la empresa, de origen británico, emitió bonds o acciones para formar el capital y se identificó a los tranvías con estos últimos (Cfr. Conde, 2004, p. 69).

13. Esta acepción no figura en el diccionario etimológico de Conde (2004), pero está muy difundida-quizá más que el significado original- en la actualidad.

14. Para este término, Musa (2005, pp. 99-100) registra la acepción 'mandarse la parte', que no se ha considerado en el trabajo, por ser entendida como fuera del uso común del término.

15. Estos resultados no pretenden ser absolutamente generalizables, sino un botón de muestra de la tesis que vertebra este trabajo y un esbozo de una posible metodología a seguir en otros estudios más especializados sobre el tema.

16. Es necesario aclarar que las acepciones de las palabras del lunfardo se presentan ordenadas -tal como se encuentran colocadas en el diccionario de Conde (2004)- según su aparición cronológica.

17. Es más, Conde (2004, p. 16) afirma que "es cosa sabida que las nuevas generaciones siempre buscan en el lunfardo más antiguo y de tanto en tanto ocurre que, después de décadas de olvido, se reflotan términos, como ha ocurrido con bondi".

\section{Bibliografía}

Arlt, R. (1998 [1929]). ¿Cómo quieren que les escriba? Aguafuertes. Obras completas. (371373). Buenos Aires: Losada.

Bordelois, I. (2006). El país que nos habla. Buenos Aires: Sudamericana.

Casas, J. (1991). Algunos italianismos en el lunfardo. E.L.U.A. 7, 27-43.

Conde, O. (2004). Diccionario etimológico del lunfardo. Bueno Aires: Taurus.

Conde, O. (3 de abril de 2009). El lunfardo y el cocoliche. Conferencia pronunciada en la Facultad de Ciencias Sociales de la UNLZ.

Conde, O. (2010). El lunfardo en la literatura argentina. Gramma. 47, 224-226. 
Esteban, J. (2002). Lenguaje lunfardo. ACTA. 17, 39-49.

Franchi, P. (2008, 9 de enero). Lunfardo. El Heraldo Hispano News (USA). http:// elheraldohispano.blogspot.com.ar/2008/01/cronicas-ciudadanas.html [Consulta 15 de febrero de 2015].

McGee, B. (s.f.). Antología lunfarda. http://www.elortiba.org/pdf/Antologia-Lunfarda.pdf [Consulta 15 de noviembre de 2013].

Montes, J. (1975). Jeringa. Buenos Aires: Corregidor.

Musa, H. (2005). Calepino. Lunfoargentino. Buenos Aires: Dunken.

Oliveto, M. (2010). La cuestión del idioma en los años veinte y el problema del lunfardo: a propósito de una encuesta en el diario Crítica. Pilken. 13, 1-9.

Picabea, M. (2004, 24 de octubre). Lunfardo siglo XXI. La lengua de los porteños, del arrabal al shopping. Clarín Sociedad. http://www.winisisonline.com.ar/tea/info/30003100/c-3078.pdf [Consulta 15 de febrero de 2015].

Pitkowski, E. (2008). Roberto Arlt revolucionario. El lunfardo de Los siete locos (Si no te interesa el título, rajá, turrito, rajá). Tinkuy. Boletín de investigación y debate. 10, 102-115. 